\title{
A Study on Image Search System using Semantics Based on Smartphone
}

\author{
Byeongtae Ahn
}

\begin{abstract}
Image semantic retrieval has been a crux to bridge "semantic gap" between the simple visual features and the abundant semantics delivered by a image. Effective image retrieval using semantics is one of the major challenges in image retrieval. We suggest a semantic retrieval and clustering method of image using image annotation user interface. And also design and implement a image semantic search management system that facilitates image management and semantic retrieval, which fully relies on the MPEG-7 standard as information base, and using a native XML database, which is Berkeley DB XML.
\end{abstract}

Keywords: Semantic, MPEG-7, Image Search, Berkeley DB, Metadata

\section{INTRODUCTION}

Image retrieval research is largely composed of two types of approaches. It is a text-based metadata image search and a content-based metadata image search. When searching for images that require accurate question expressions, the text-based approach has limitations compared to content-based search when it is difficult to extract clear feature questions. Therefore, to solve this problem, we propose a content-based image retrieval technique using semantics.

The content-based image retrieval technique is easy to understand and is suitable for expressing a simple and flexible standardized approach in a meaningful expression. MPEC-7 was used to solve this efficient technique. MPEC-7 enables efficient search by describing standardized image semantic information in metadata for multimedia objects, and by using metadata, it can represent special features of the body such as title, author, image content, and color histogram of the descriptor. Therefore, this paper is very effective in image retrieval system based on embedded database in mobile environment based on MPEC-7 standard. And in this paper, we designed and implemented an image semantic search management system in a mobile environment. The image semantic search management system uses MPEG-7 to improve search efficiency through the image search automatic feature extraction module, and uses Berkeley DB XML, an embedded database system, for optimized management of image search. And by supporting various language searchers, any language can be used like a standard language. The

Manuscript received on June 16, 2021.

Revised Manuscript received on June 18, 2021.

Manuscript published on June 30, 2021.

* Correspondence Author

Byeongtae Ahn*, Liberal \& Arts College, Anyang University, Anyang-shi, South Korea. Email: ahnbt@anyang.ac.kr

(c) The Authors. Published by Blue Eyes Intelligence Engineering and Sciences Publication (BEIESP). This is an open access article under the CC BY-NC-ND license (http://creativecommons.org/licenses/by-nc-nd/4.0/) currently implemented system supports English, Chinese, and

In Chapter 2 of this paper, related studies are examined, in Chapter 3, a clustering technique for image semantic retrieval is proposed, and in Chapter 4, an image semantic retrieval system is designed. In Chapter 5, an image semantic search system is implemented, and in Chapter 6 proposes conclusions and future tasks.

\section{RELATED WORK}

MPEG-7 document storage methods are classified into pure XML database storage methods and XML-enabled database storage methods [1]. The big difference between them is in the format of the data model they use. The former is based on XML data models such as the Document Object Model (DOM) and Object Exchange Model (OEM), and the latter is based on the traditional relational data model or object-oriented data model, and each has its own advantages and disadvantages. In order to express complex types of MPEG-7 schema using an XML capable database, modification of the database structure is inevitable [2]. This structural modification makes programming difficult and incurs significant system overhead. Therefore, in recent years, many studies have been conducted using a pure XML database storage method for the management of MPEG-7 documents.

In the pure XML database storage method, the types are divided by the size of the record, which is the smallest unit to be stored in the XML document. Element-based (EB), subtree-based (SB), and document-based ( DB). The Lore system or TIMBER follows the alignment-based policy. Each element is used as a storage unit, and the element is assigned a number in advance and stored [3[[4]]. Natix uses a subtree-based policy, and stores the entire XML document in the form of a subtree according to the size of the physical storage page [5]. Apache Xindice uses a DB policy that organizes and stores the entire XML document into a single record. However, since these systems do not consider schema, it is difficult to efficiently utilize schema information when searching or managing [6]. On the other hand, if you store and manage XML data using schema information, you can utilize various storage policies. In addition, when constructing an index, a schema-based path index can be used, and validity can be verified in advance for query processing and updating. OrientX is a representative system [7].

This system uses various storage policies based on schema information. In particular, we propose each clustering method based on element-based and subtree-based. These clustering methods can reduce storage space and reduce I/O time. However, this method was developed for general XML document management, and there are many insufficient points to support a schema of a complex structure such as MPEG-7.

\section{Published By:}

Blue Eyes Intelligence Engineering and Sciences Publication

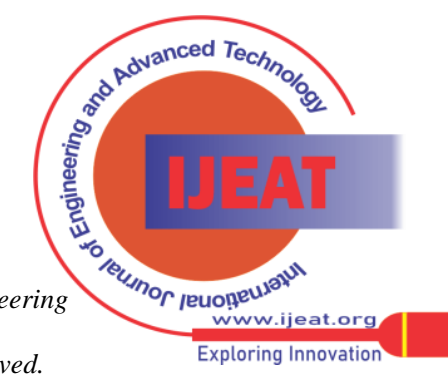


MPEG-7 is a structure that can derive various types from an abstract type, and since it has an organic association between each element, it must be saved in consideration of this. This is because if the clustering policy of MPEG-7 documents is used based on the cardinality of the XML schema as in OrientX, the system performance can be degraded by increasing the number of block accesses on the disk.

Therefore, in this paper, we propose a fragment clustering method that considers the characteristics of MPEG-7.

\section{CLUSTERING STORAGE METHOD}

The clustering storage technique proposed in this paper is stored using segmented fragments, and its components become XML instance documents generated by Fine-Grained expression. Fragments largely have identification ID, name, and type. Through the analysis of the MPEG-7 schema, related elements are represented as fragments of the same type and are placed in the same block through a clustering process.

\subsection{Mpeg-7 schema redefinition}

We were able to grasp the relationship between each element through analysis of various MPEG-7 applications [8]. Correlation information of elements can be represented by a method of redefining the schema at the MPEG-7 schema level. In other words, when defining the elements of the MPEG-7 schema, R-CT properties indicating that there is a relationship between related elements are given, and those having relationships are represented as fragments of the same type, and these are clustered and stored. This can greatly improve the query processing speed because it is possible to process queries by accessing the same block for queries of closely related elements that occur frequently in MPEG-7 applications[9].

Fig. 1 is an MPEG-7 schema that redefines R-CT (Relationship-Clustering Type) properties, and shows that the $<$ MovingRegion $>$ element and the $<$ ViedoSegment $>$ element are related to each other.

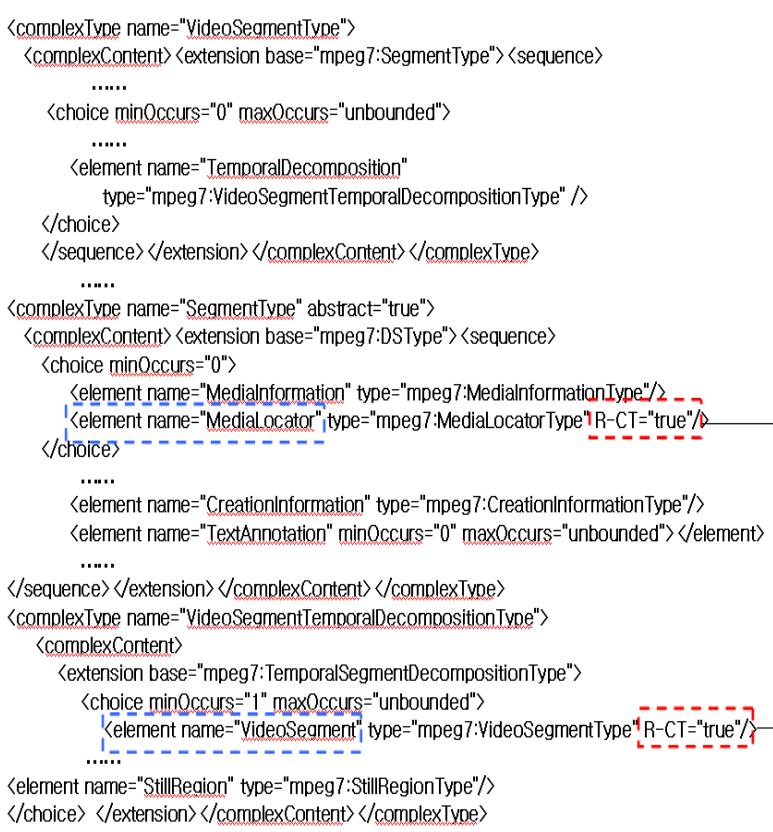

〈element name="StillRegion" type="mpeg7:StillRegionType" $/>$

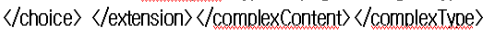

Fig 1. MPEG-7 schema redefinition

\subsection{Fragment}

In order to fragment an MPEG-7 document, a fragment model must first be constructed through MPEG-7 schema analysis. In the case of Figure 1, the <MovingRegion> element and the $<$ VideoSegment $>$ element are related to each other. Therefore, fragments composed of two elements are of the same type, and when expressed as a fragment model, they are formed as a group as shown in Fig2.

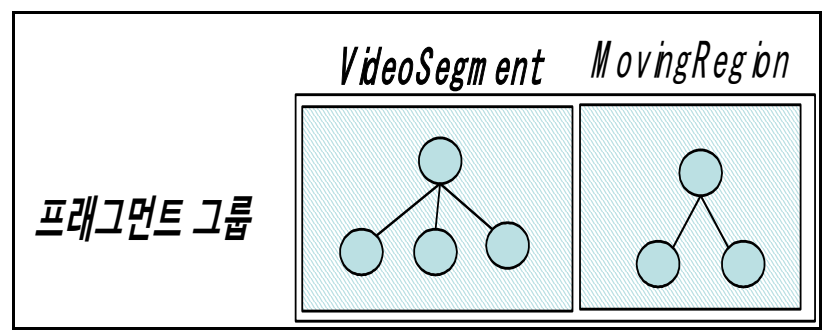

Fig. 2. Fragment structure

\subsection{Clustering}

It is desirable to store all fragments in one block, but considering the limited block size, this is impossible. Therefore, we classify the fragment type and store the block in clustering. However, MPEG-7 application systems can use various queries in the application. Therefore, even if the fragments are not of the same type, there is a case where they must be placed in one block. For this, we use a fragment clustering technique. Fig3. shows how fragments are actually stored in a block[10].

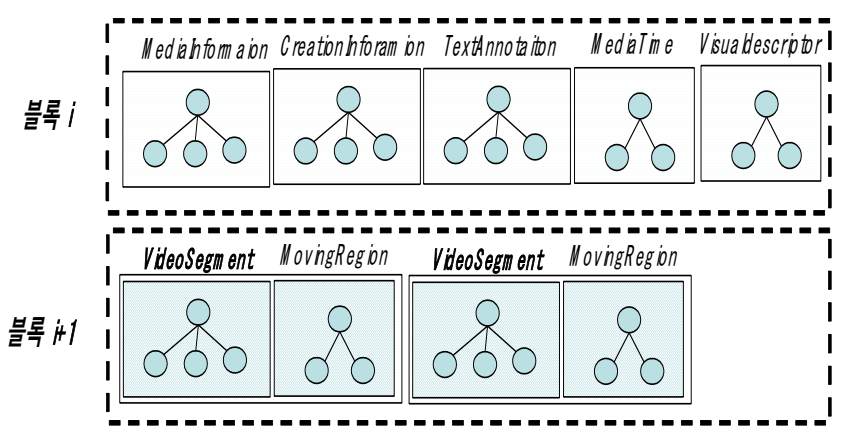

Fig. 3. Block storage type of fragment

When such fragment clustering is performed, the query can obtain the desired result with a single block input/output.

a) When using KeyImage to find a specific image for a video.

b) When searching for KeyFrame information corresponding to each video segment.

In contrast, OrientX, which uses a similar method, only supports clustering in which repeated elements are stored in the same block considering only the cardinality of the element. Therefore, in OrientX, the speed is slowed down because the other two blocks have to be accessed in order to obtain the desired result for the previous query[11].

Published By:

Blue Eyes Intelligence Engineering and Sciences Publication

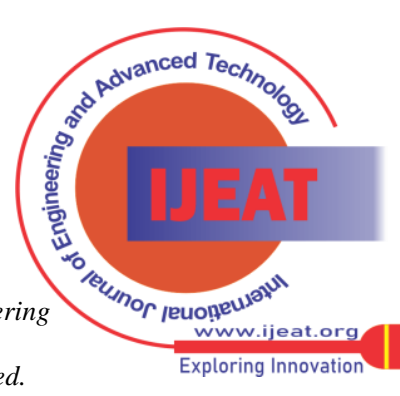




\section{SYSTEM DESIGN}

Most image search management systems have limited support for semantic search. In this paper, we designed a new image semantic search management system that supports semantic retrieval based on MPEG-7. Recently, many image search management systems have been developed based on MPEG-7 technology. In particular, this is the case where MIRROR (MPEG-7 Image Retrieval Refinement based On Relevance feedback), CIRES (Content based Image Retrieval System), and NETRA (A toolbox for navigating large image databases) are typically used. However, most of them also support semantic search with extremely limited support[12]. Therefore, in this paper, we designed a new image semantic retrieval management system based on MPEG-7 that efficiently supports semantic retrieval. This system reinforces the image semantic retrieval function based on the contents that are interchangeable with standard MPEG-7 multimedia technology. This system supports multimedia contents, metadata, automatic recognition of semantic descriptors, and MPEG-7 and XML encoding. And this system provides three types of types. It is a direct information search in Berkeley $\mathrm{DB}$, a content-based information search, and a semantic search of image information. In particular, the semantic retrieval technology is the main characteristic of this system and supports an efficient retrieval system through an interface capable of supporting various languages and image semantic retrieval[13].

The following system consists of an image annotation subsystem, an image search subsystem, and a multimedia database. All annotation information about the image is converted into MPEG-7 descriptor and stored in MPEG-7 DB. The image search sub-system performs MPEG-7 document search using a search engine and displays the search results. All annotations on the image generate descriptive information and are stored as MPEG-7 values. The stored images are matched with MPEG-7 documents by the multimedia database system and stored in the image database. The image annotation subsystem consists of text, meaning, metadata, and automatically generated visualization annotations. The MPEG-7 generation module will get an MPEG-7 metadata instance through the MPEG-7 schema, and the instance document is saved as Berkeley DB XML. The saved content is displayed as a view through an interface through the XML File Navigatotr. The image search subsystem consists of three elements: a search panel, a search engine, and a result view panel. The search engine module receives the user query from the search panel and translates the user query in XQuery by the XQuery constructor[14].

Berkeley DB XML is a dedicated XML database system that allows access to documents stored in a container based on XQuery. The result of query processing is generated in XML format in Berkeley DB XML. The image search sub-system provides the following three methods as a search method for image matching. The first is search by text input technology. This search is based on keyword matching of text metadata using a text query, and while it is simple and easy, it has the disadvantage of requiring the user to directly enter the desired search keyword. The second is search by content-based technology. This search searches for images using two visual descriptors ColorLayout and ScalableColor for a content-based image search. The ColorLayout descriptor is used for high-speed image search, and the ScalableColor descriptor applies the value of the color histogram to search. The third is search by similar semantic description. This semantic search mechanism supports semantic queries based on MPEG-7 semantic descriptors. In the semantic search interface, three objects and two relationships were defined to implement semantic search research[15].

\section{SYSTEM IMPLEMENTATION}

The image semantic retrieval system was implemented in a mobile environment, and a highly portable JAVA based on MS Window XP was used as the development environment.

\subsection{Annotation User Interface}

The image semantic search management system provides users with an easy-to-use annotation user interface. Fig4 shows the image annotation user interface.

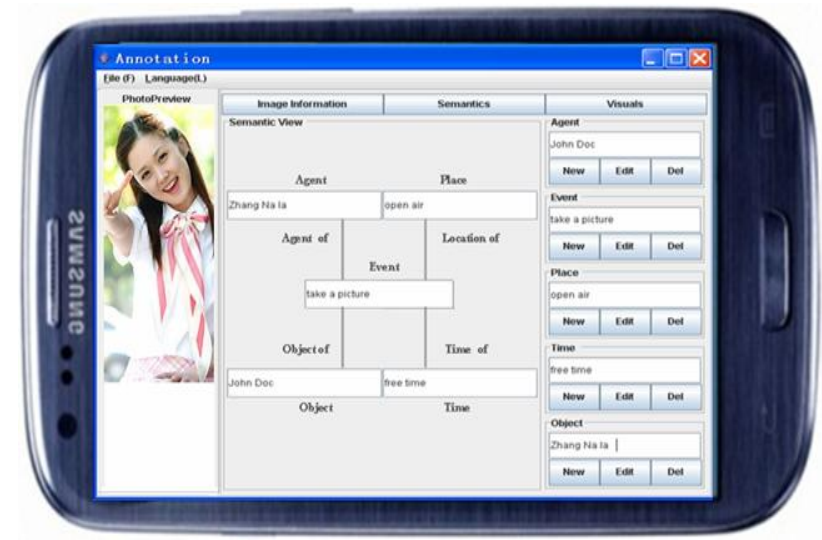

Fig. 4. Image annotation user interface

The image annotation user interface displays images, technical documents, semantic annotation content for MPEG-7 metadata instances, and so on. In the semantic annotation panel of the image semantic search management system, semantic object contents such as user agent, event, place, and time are described. Semantic objects and relationships appear on the semantic panel, and semantic annotations are generated as appropriate XML files.

\subsection{MPEG-7 file for semantic information}

The metadata of MPEG-7 is an XML document based on XML Schema. Figure 8 shows the semantic information as an MPEG-7 file.

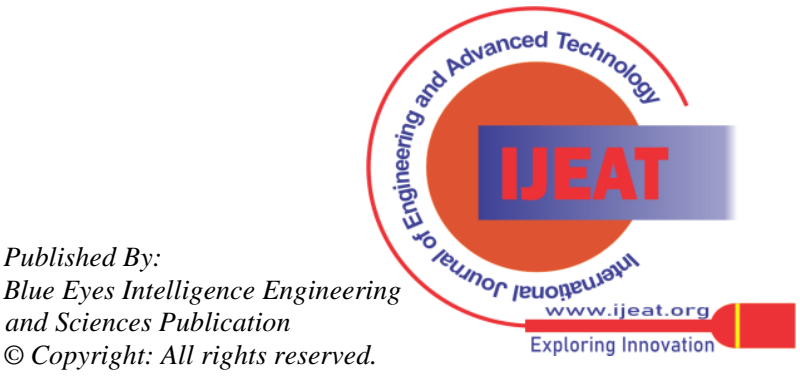




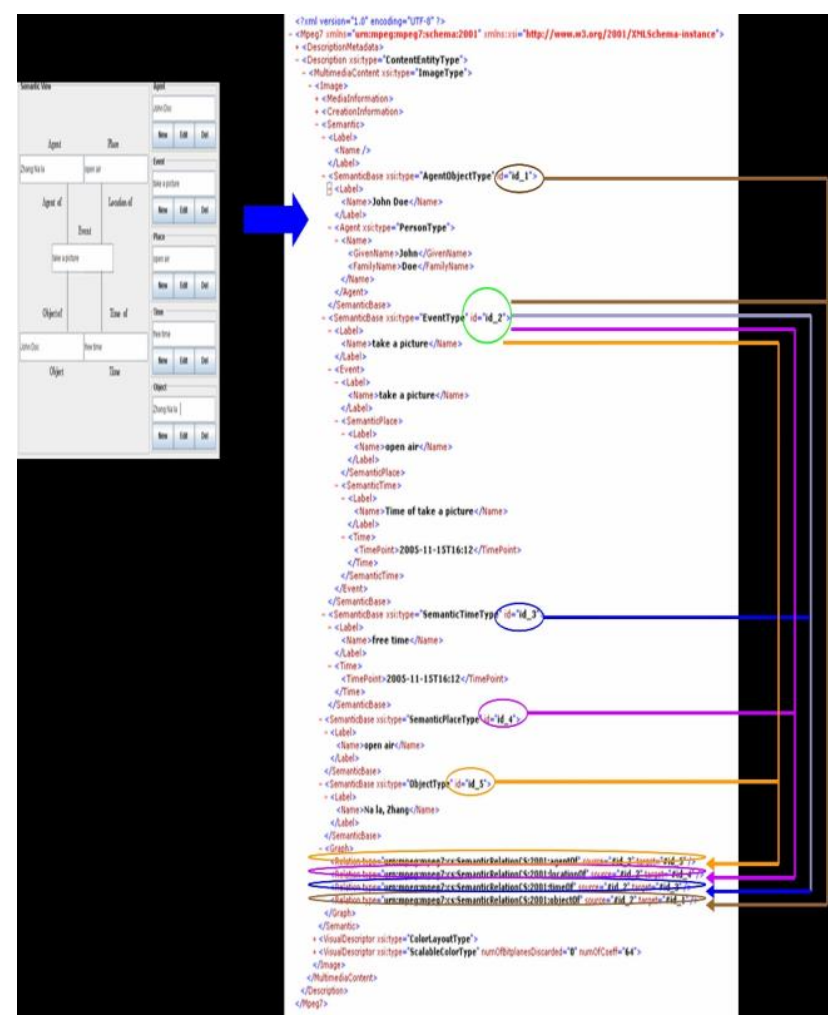

Fig 5. MPEG-7 file for semantic information

Fig5. shows the MPEG-7 technology in the semantic field from the semantic annotation input by the user. Some of the contents of the file describe the tags <AgentObject>, $<$ Event $>$, $<$ SemanticTime $>$, $<$ SemanticPlace $>$, and $<$ Object $>$ and the relationship between them.

\subsection{Search user interface}

The image search sub-system provides the user with a search function with photo annotations. Fig6. shows the image search user interface.

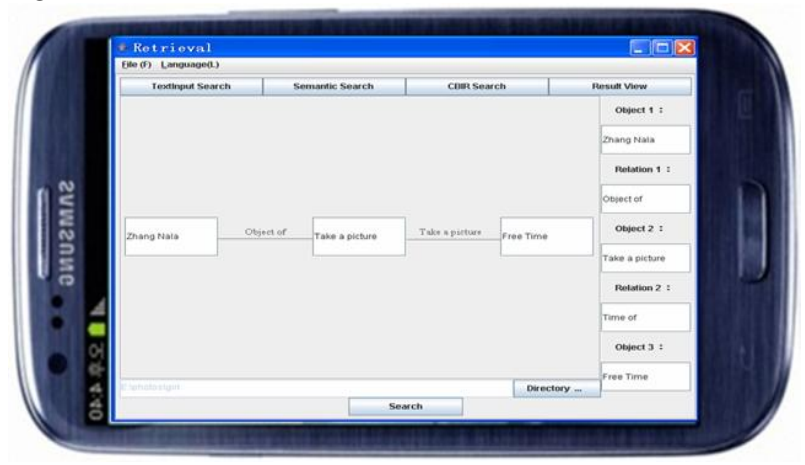

Fig 6. Image search user interface

The image semantic search management system provides a search mechanism for semantic technology search. The meaning search using the graph starts, and the user's manual for information input appears on the right side of the interface. The graph shown in the figure above is defined by three objects and two relationships. The search checks whether it structurally matches the given graph, and then indicates that it matches all documents that contain semantic objects that are the same as relations in Berkeley DB XML.

\subsection{Image search process}

Fig7. is a schematic diagram of the image search process.

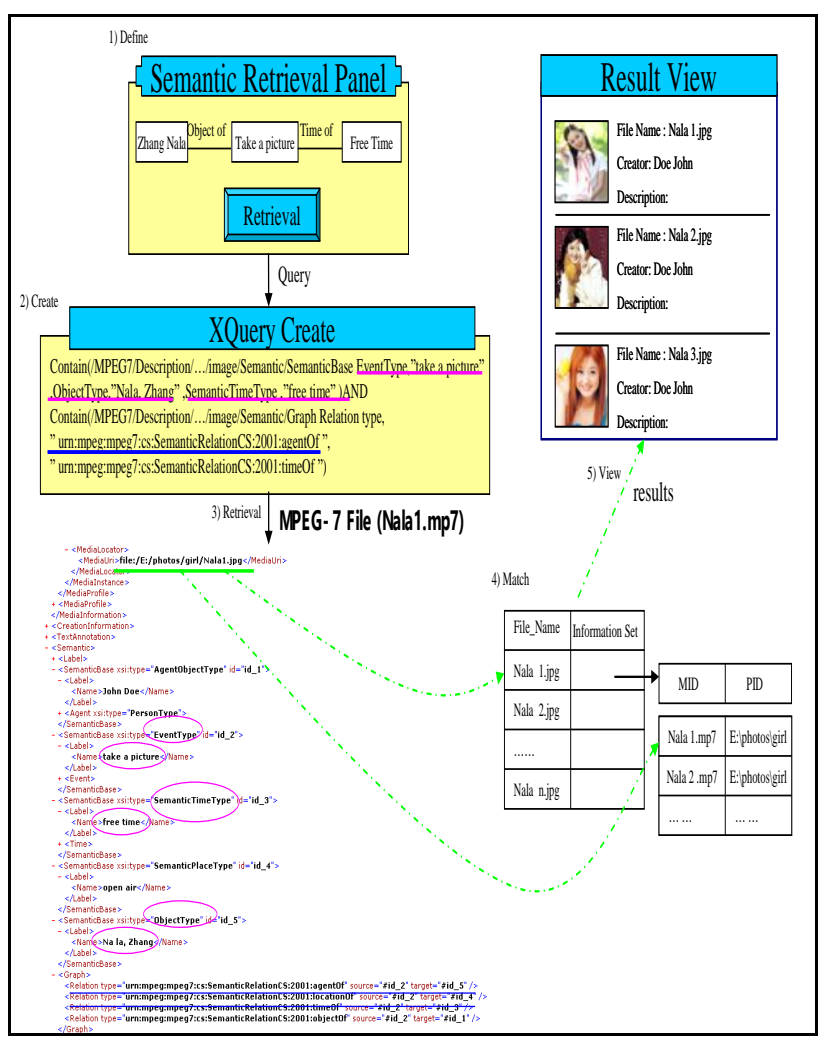

Fig. 7. Image search process

The image search process consists of a total of five steps: defining the search process, generating XQuery, searching MPEG-7 files, matching photos, and viewing results.

1) Definition of search process

The image search subsystem provides an excellent search user interface that enables easy and direct query detailing for users.

2) XQuery creation

After we define the retrieval process, the XQuery generated by the XQuery generator retrieves all MPEG-7 documents in Berkeley DB that contain semantic objects after retrieval.

3) MPEG-7 file search

After creating an XQuery, the matching MPEG-7 file is searched from the database according to the search process

4) Match the photo

Matching the image is implemented through MPEG-7 files. If File_Name and MPEG ID match, an image result is obtained.

5)View the result

The image result is displayed on the screen.

\section{CONCLUSION AND FUTURE WORKS}

We have shown that a more efficient fragment clustering method is possible by expressing the property of R-CT in the MPEG-7 schema. And based on this, we proposed a storage system (MDSS) based on an embedded XML DB for storing MPEG-7 documents.

Published By:

Blue Eyes Intelligence Engineering and Sciences Publication

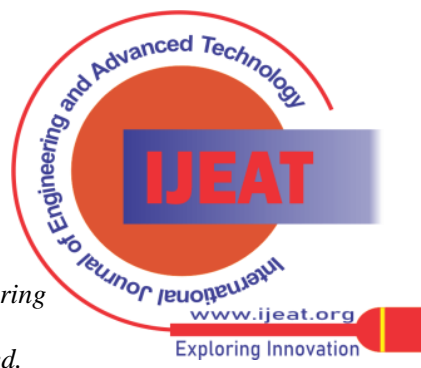


The fragment clustering method devised through this study analyzes the MPEG-7 schema to fragment the MPEG-7 $\mathrm{XML}$ document and stores the relevant ones in the same container block in the lower Berkeley DB XML to efficiently search for MPEG-7 documents. I can. In addition, in the proposed MDSS, the validity of a query can be verified in advance by the MPEG-7 schema, and it can efficiently cope with the document update. The schema management subsystem of the current system has been developed. And for clustering, a clustering management sub-system is being implemented.

\section{REFERENCES}

1. The image semantic search management system provides a set of effective image management and automatic feature extraction modules of the search system. In addition, traditional feature extraction based on the MPEG-7 standard information base was added.

2. MIRROR is a platform for image retrieval research and development based on content using MPEG-7 technology. The system can easily add new images to the database with an improved modular approach and a new structured approach.

3. CIRES extracts features of an effective integrated method, and the search method can query various query images, which queries in a format different from the traditional method.

4. NETRA queries in a weighted integrated method of shape, color, and text characteristics. This system is effective for content-based search. In particular, it is possible to efficiently search for images based on color, structure, and text content.

5. W.-K. Chen, Linear Networks and Systems. Belmont, Calif.: Wadsworth, pp. 123-135, 1993. (Book style)

6. H. Poor, "A Hypertext History of Multiuser Dimensions," MUD History, http://www.ccs.neu.edu/home/pb/mud-history.html. 1986. (URL link *include year)

7. K. Elissa, "An Overview of Decision Theory," unpublished. (Unplublished manuscript)

8. R. Nicole, "The Last Word on Decision Theory," J. Computer Vision, submitted for publication. (Pending publication)

9. C. J. Kaufman, Rocky Mountain Research Laboratories, Boulder, Colo., personal communication, 1992. (Personal communication)

10. D.S. Coming and O.G. Staadt, "Velocity-Aligned Discrete Oriented Polytopes for Dynamic Collision Detection," IEEE Trans. Visualization and Computer Graphics, vol. 14, no. 1, pp. 1-12, Jan/Feb 2008, doi:10.1109/TVCG.2007.70405. (IEEE Transactions )

11. S.P. Bingulac, "On the Compatibility of Adaptive Controllers," Proc. Fourth Ann. Allerton Conf. Circuits and Systems Theory, pp. 8-16, 1994. (Conference proceedings)

12. H. Goto, Y. Hasegawa, and M. Tanaka, "Efficient Scheduling Focusing on the Duality of MPL Representation," Proc. IEEE Symp. Computational Intelligence in Scheduling (SCIS '07), pp. 57-64, Apr. 2007, doi:10.1109/SCIS.2007.367670. (Conference proceedings)

13. J. Williams, "Narrow-Band Analyzer," PhD dissertation, Dept. of Electrical Eng., Harvard Univ., Cambridge, Mass., 1993. (Thesis or dissertation)

14. E.E. Reber, R.L. Michell, and C.J. Carter, "Oxygen Absorption in the Earth's Atmosphere," Technical Report TR-0200 (420-46)-3, Aerospace Corp., Los Angeles, Calif., Nov. 1988. (Technical report with report number)

15. L. Hubert and P. Arabie, "Comparing Partitions," J. Classification, vol. 2, no. 4, pp. 193-218, Apr. 1985. (Journal or magazine citation)

\section{AUTHORS PROFILE}

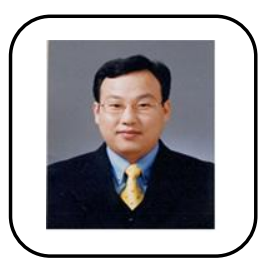

Byeogtae Ahn, is a Faculty of Division of Liberal Arts at Anyang University, Korea. His research interests include: Image Processing, Video Analysis, IoT, BlockChain, Multimedia Database and MPEG-7. His address is: 37-22, Samduck Minahn-gu Anyang-City Gyeonggi-do, 430-714 South Korea. His phone number is $+82-31-463-1204$ and the email address is

ahnbt@anyang.ac.kr

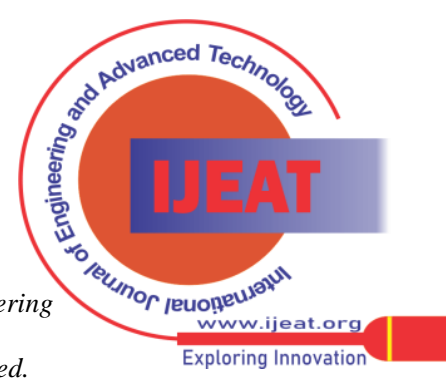

